\title{
COLOR ENERGY OF A UNITARY CAYLEY GRAPH
}

\author{
Chandrashekar Adiga, E. Sampathkumar \\ AND \\ M.A. SRIRAJ ${ }^{1}$ \\ Department of Studies in Mathematics \\ University of Mysore \\ Manasagangotri \\ Mysore - 570 006, India \\ e-mail: c_adiga@hotmail.com \\ esampathkumar@gmail.com \\ srinivasa_sriraj@yahoo.co.in
}

\begin{abstract}
Let $G$ be a vertex colored graph. The minimum number $\chi(G)$ of colors needed for coloring of a graph $G$ is called the chromatic number. Recently, Adiga et al. [1] have introduced the concept of color energy of a graph $E_{c}(G)$ and computed the color energy of few families of graphs with $\chi(G)$ colors. In this paper we derive explicit formulas for the color energies of the unitary Cayley graph $X_{n}$, the complement of the colored unitary Cayley graph $\overline{\left(X_{n}\right)_{c}}$ and some gcd-graphs.
\end{abstract}

Keywords: coloring of a graph, unitary Cayley graph, gcd-graph, color eigenvalues, color energy.

2010 Mathematics Subject Classification: 05C15, 05C25, 05 C50.

\section{REFERENCES}

[1] C. Adiga, E. Sampathkumar, M.A. Sriraj and A.S. Shrikanth, Color energy of a graph, Proc. Jangjeon Math. Soc. 163 (2013) 335-351.

[2] N. Biggs, Algebraic Graph Theory, Second Edition (Cambridge Mathematical Library, Cambridge University Press, 1993).

\footnotetext{
${ }^{1}$ The first author is thankful to the university grants commission Goverment of India for the financial support under the grant F.510/2/SAP-DRS/2011. The second and third authors are thankful to DST for its financial support under the project SR/S4/MS 236/04.
} 
[3] C. Godsil and G. Royle, Algebraic Graph Theory (Graduate Texts in Mathematics, Springer, 207, 2001).

[4] I. Gutman, The energy of a graph, Ber. Math. Stat. Sekt. Forschungsz. Graz 103 (1978) 1-22.

[5] G.H. Hardy and E. M. Wright, An Introdution to Theory of Numbers, Fifth Ed. (Oxford University Press New York, 1980).

[6] W. Klotz and T. Sander, Some properties of unitary Cayley graphs, Electron. J. Combin. 14 (2007) \#R45.

[7] A. Ilić, The energy of unitary Cayley graphs, Linear Algebra Appl. 431 (2009) 18811889. doi:10.1016/j.laa.2009.06.025

[8] M. Mollahajiaghaei, The eigenvalues and energy of integral circulant graphs, Trans. Combin. 1 (2012) 47-56.

[9] E. Sampathkumar and M.A. Sriraj, Vertex labeled/colored graphs, matrices and signed graphs, J. Combin. Inform. System Sci., to appear.

[10] W. So, Integral circulant graphs, Discrete Math. 306 (2006) 153-158. doi:10.1016/j.disc.2005.11.006

Received 31 January 2013 Revised 17 September 2013 Accepted 16 October 2013 